\title{
Further Results on Stability Analysis for Uncertain Delayed Neural Networks with Reliable Memory Feedback Control
}

\author{
Wenhao Wang, ${ }^{1}$ Xiao Cai, ${ }^{2}$ Kaibo Shi $\mathbb{D},{ }^{3}$ Hong Zhu, ${ }^{1}$ and Shouming Zhong $\mathbb{D}^{4}$ \\ ${ }^{1}$ School of Automation Engineering, University of Electronic Science and Technology of China, Chengdu, Sichuan 611731, China \\ ${ }^{2}$ School of Information and Software Engineering, University of Electronic Science and Technology of China, Chengdu, \\ Sichuan 611731, China \\ ${ }^{3}$ School of Electronic Information and Electrical Engineering, Chengdu University, Chengdu, Sichuan 610106, China \\ ${ }^{4}$ School of Mathematics Sciences, University of Electronic Science and Technology of China, Chengdu, Sichuan 611731, China \\ Correspondence should be addressed to Kaibo Shi; skbs111@163.com
}

Received 15 December 2020; Revised 27 January 2021; Accepted 18 February 2021; Published 24 March 2021

Academic Editor: Huaiqin $\mathrm{Wu}$

Copyright (c) 2021 Wenhao Wang et al. This is an open access article distributed under the Creative Commons Attribution License, which permits unrestricted use, distribution, and reproduction in any medium, provided the original work is properly cited.

\begin{abstract}
This study proposes further results for the stability analysis issue of uncertain delayed neural networks (UDNNs) via the reliable memory feedback control scheme. First, an improved quadratic function method is introduced for constructing a novel term $V_{1}(x(t))$, which can fully excavate some intrinsic relationships between the delay derivative information and time-delay information. Based on the time-delay-product function (TDPF) and linear convex combination method (LCCM), the information storage is further improved for obtaining new theoretical results. Second, by using resultful integral inequalities and correlation analysis approaches, several relaxed criteria are established with respect to the asymptotical stability of the considered UDNNs. Third, the desired reliable memory feedback controller (RMFC) is achieved, which can ensure the system stability of UDNNs. Lastly, two numerical experiments are given to illustrate the significance of the theoretical results.
\end{abstract}

\section{Introduction}

As we all know, because the structure of the NN model is similar to the synapse structure of the human brain, it can be described by a variety of differential equations [1-7]. The wide application of $\mathrm{NNs}$ in various fields has received widespread attention, such as signal processing [8], fault diagnosis [9], optimization problem solving [10], pattern recognition [11], image processing [12], and other fields [13-16]. However, artificial NNs always need to be maintained in practical engineering applications, so the stability of NNs has also been extensively studied by scholars at home and abroad [15, 17-19]. In the engineering application of NNs, the signal transmission between synapses has a time delay, and this delay may lead to instability of the NNs, increased oscillation, or performance degradation [20-22]. Therefore, the stability research of time-varying neural networks (DNNs) has also received extensive attention [23-26]. Compared with NNs, the latter requires more technical means and engineering requirements to maintain stability in technical analysis [27-29]. Therefore, the research on DNNs is obviously more important.

Thus, in order to effectively solve this problem, Lyapunov-Krasovskii functional (LKF) method is proposed $[18,30-33]$. So far, many researchers have made a lot of contributions of how to establish a suitable LKF in order to better study the delayed systems [13, 34-37]. In [30], the authors proposed a novel LKF, which contains a common double-integral term, an augmented double-integral term, and two delay-product-type terms, was constructed to analyze the exponential stability. In [31,32], the reciprocally convex matrix inequality was an important technique to develop stability criteria for the systems with a time-varying delay which was studied. In $[18,33,35]$, based on some effective integral inequalities, the integral term in LKF was reasonably scaled. In [34], the authors first proposed robust $H_{\infty}$ control for T-S fuzzy systems with state and input timevarying delays via DTPF. In [13], the authors proposed novel 
weighting-delay-based stability criteria for system research. In $[36,37]$, the authors mainly studied the sampled-data control and gave the analysis and proof of related stability.

However, there are many methods to construct a reasonable LKF, but only increasing the cross-sectional area will hardly improve, and it will cause a heavy calculation burden $[23,38,39]$. Therefore, the method of constructing LKF from a new perspective has become a hot issue in current research $[34,40]$. Through in-depth study of existing work, this paper proposes an improved DTPF strategy to construct a new LKF, which fully considers information concerning time delays and the derivative information of both states and time delays, and the conservativeness of the guidelines can be further reduced. The issues discussed above have inspired the purpose of this study.

Based on the above discussions, we establish some new stability criteria for UDNNs, and a reliable memory feedback controller is designed to ensure that the considered system is asymptotically stable. Compared with the existing results [22-25], the main contributions of this paper are as follows:

(1) A novel quadratic function $V_{1}(x(t))$ is constructed via developing an improved TDPF approach, which can fully excavate some intrinsic relationships between the delay derivative information and the time delay

(2) Based on this construction method of LKF, the information storage performance of the function is strengthened, an appropriate integral inequality and linear convex combination method are adopted, and a more conservative stability criterion is obtained

(3) Different from the earlier work, this paper designs a new RMFC, which fully considers the effective transmission of the three state signals of the controller while enhancing the performance of the controller

\section{Preliminaries}

Consider the following UDNN system:

$$
\begin{aligned}
\dot{z}(t)= & -\mathscr{A}(t) z(t)+\mathscr{W}_{0} g(z(t)) \\
& +\mathscr{W}_{1} g(z(t-h(t)))+u(t)+a,
\end{aligned}
$$

where $z(t)=\left[z_{1}(t), z_{2}(t), \ldots, z_{n}(t)\right]^{T} \in \mathbb{R}^{n}$ denotes the neuron state vector and $u(t)$ is the control input signal. $g(\cdot)=\left[g_{1}(\cdot), g_{2}(\cdot), \ldots, g_{n}(\cdot)\right]^{T} \in \mathbb{R}^{n}$ represents the neuron activation function; $\mathscr{A}(t)=\mathscr{A}+\Delta \mathscr{A}(t)$, and $\Delta \mathscr{A}(t)$ is the revalued unknown matrix representing time-varying parameter uncertainties of $(1)$ and satisfies $\Delta \mathscr{A}(t)=\mathscr{H} \mathscr{F}(t) \mathrm{E}$. Here, $\mathscr{A}>0$ is a symmetric matrix; $H, \mathscr{E}$ are known real constant matrices of appropriate dimensions. $\mathscr{F}(t) \in R^{l_{1} \times l_{2}}$ is an unknown time-varying matrix function satisfying $\mathscr{F}^{T}(t) \mathscr{F}(t) \leq \mathscr{I} . \mathscr{W}_{0}$ indicates the connection weight matrix, and $\mathscr{W}_{1}$ expresses the delayed connection weight matrix; $a=\left[a_{1}, \ldots, a_{n}\right]^{T} \in \mathbb{R}^{n}$ is a vector. $h(t)$ is time varying and satisfies

$$
\begin{aligned}
& 0 \leq h(t) \leq h, \\
& 0 \leq \dot{h}(t) \leq \mu .
\end{aligned}
$$

Based on Assumption 1 in [25], suppose that $z^{*}$ is the balance point of UDNN (1), which can be transferred to the origin by conversion, $x(\cdot)=z(\cdot)-z^{*}$. Then, system (1) can be expressed as

$$
\dot{x}(t)=-\mathscr{A} x(t)+\mathscr{W}_{0} f(x(t))+\mathscr{W}_{1} f(x(t-h(t)))+u(t),
$$

where $x(\cdot)=\left[x_{1}(\cdot), x_{2}(\cdot), \ldots, x_{n}(\cdot)\right]^{T} \in \mathbb{R}^{n}$ is the state vector of the transformed system and $f(\cdot)=\left[f_{1}(\cdot), \ldots, f_{n}(\cdot)\right]^{T} \in \mathbb{R}^{n}$ with $f_{j}\left(x_{j}(\cdot)\right)=g_{j}\left(x_{j}(\cdot)+\right.$ $\left.z_{j}^{*}\right)-g_{j}\left(z_{j}^{*}\right)$ being the activation function of the converted system. Based on (3), it holds that

$$
\begin{aligned}
l_{i}^{-} & \leq \frac{g_{i}\left(u_{i}+y_{i}^{*}\right)-g_{i}\left(y_{i}^{*}\right)}{u_{i}+y_{i}^{*}-y_{i}^{*}}=\frac{f_{i}\left(u_{i}\right)}{u_{i}} \\
& \leq l_{i}^{+}, \quad f_{i}(0)=0, \forall u \neq 0, i=1, \ldots, n .
\end{aligned}
$$

In order to express a reliable control problem, the following actuator failure models are used in this technical description: for $m=1, \ldots, \vartheta, n=1, \ldots, v$ :

$$
u_{m n}^{F}(t)=\left(1-\pi_{m}^{n}\right) u(m)(t), \quad 0 \leq \underline{\pi}_{m}^{n} \leq \pi_{m}^{n} \leq \bar{\pi}_{m}^{n},
$$

where $\pi_{m}^{n}$ is an unknown constant. Here, $n$ is the $n$th case of failure, and $j$ is the total number of failure cases. $u_{m n}^{F}(t)$ indicates the control signal from the signal of the $m$ th actuator in the $n$th fault situation. $\underline{\pi}_{m}^{n}$ and $\bar{\pi}_{m}^{n}$ represent the lower and upper bounds of $\pi_{m}^{n}$. First, when $\underline{\pi}_{m}^{n}=\bar{\pi}_{m}^{n}=0$, in the $n$th failure mode, the $m$ th actuator has no failure. When $\underline{\pi}_{m}^{n}=\bar{\pi}_{m}^{n}=1$, the $m$ th actuator $u_{m}$ stops working in the $n$th fault situation. When $0 \leq \underline{\pi}_{m}^{n} \leq \bar{\pi}_{m}^{n}<1$, in the $n$th fault condition, the type of actuator failure is a gradual descent condition.

The actuator fault matrix $\Pi$ is designed as the following:

$$
0 \leq \underline{\Pi}=\operatorname{diag}\left\{\underline{\pi}_{1}^{n}, \ldots, \underline{\pi}_{m}^{n}\right\} \leq \Pi \leq \bar{\Pi}=\operatorname{diag}\left\{\bar{\pi}_{1}^{n}, \ldots, \bar{\pi}_{m}^{n}\right\} \leq I_{i} .
$$

Next, we denote the following notation:

$$
\begin{aligned}
& \tilde{\pi}_{a m}^{n}=\frac{\bar{\pi}_{m}^{n}+\underline{\pi}_{m}^{n}}{2}, \quad \Pi_{a}=\operatorname{diag}\left\{\tilde{\pi}_{a 1}^{n}, \ldots, \tilde{\pi}_{a m}^{n}\right\}, \\
& \tilde{\pi}_{b m}^{n}=\frac{\bar{\pi}_{m}^{n}-\underline{\pi}_{m}^{n}}{2}, \quad \Pi_{b}=\operatorname{diag}\left\{\tilde{\pi}_{b 1}^{n}, \ldots, \tilde{\pi}_{b m}^{n}\right\} .
\end{aligned}
$$

From (7) and (8), $\Pi$ can be expressed as follows:

$$
\Pi=\Pi_{a}+\Pi_{b} \Delta \Pi_{0}
$$

where $\left\|\Delta \Pi_{0}\right\| \leq I_{m}$.

Thus, we get the reliable controller design as follows:

$$
u^{F}(t)=(\mathscr{I}-\Pi) u(t)
$$


Remark 1. Compared with the current design methods of the reliability controller [26-29], this paper introduces the RMFC design with effective lowering of the brake. The reliability control design considered in (10) is more comprehensive than the general reliability control design, which has a wider range of applications. Then, the RMFC is as follows:

$$
u(t)=\mathscr{K}_{1} x(t)+\mathscr{K}_{2} x(t-h(t))+\mathscr{K}_{3} x(t-h) .
$$

From the above discussion, consider combining (10) and (11) to get the reliability controller design as follows:

$$
\begin{aligned}
u^{F}(t)= & (\mathscr{I}-\Pi) \mathscr{K}_{1} x(t)+(\mathscr{I}-\Pi) \mathscr{K}_{2} x(t-h(t)) \\
& +(\mathscr{I}-\Pi) \mathscr{K}_{3} x(t-h) .
\end{aligned}
$$

(3) with controller (12) can be represented as

$$
\begin{aligned}
\dot{x}(t)= & \left(-\mathscr{A}(t)+(\mathscr{I}-\Pi) \mathscr{K}_{1}\right) x(t)+\mathscr{W}_{0} f(x(t)) \\
& +\mathscr{W}_{1} f(x(t-h(t))) \\
& +(\mathscr{I}-\Pi) \mathscr{K}_{2} x(t-h(t))+(\mathscr{I}-\Pi) \mathscr{K}_{3} x(t-h) .
\end{aligned}
$$

Lemma 1 (see [38]). Given a symmetric positive definite matrix $\mathscr{R}$, scalar $\alpha$, scalar $\beta$, and $\alpha<\beta$, and $e$ in $[\alpha, \beta] \longrightarrow \mathbb{R}^{n}$. The following are the inequalities under the given conditions:

$$
\begin{aligned}
& \left\{\begin{array}{l}
(\beta-\alpha) \int_{\alpha}^{\beta} \dot{e}^{T}(s) \mathscr{R} \dot{e}(s) \mathrm{d} s, \quad \geq \chi_{1}^{T} \mathscr{R} \chi_{1}, \\
(\beta-\alpha) \int_{\alpha}^{\beta} e^{T}(s) \mathscr{R} e(s) \mathrm{d} s, \quad \geq \hat{\chi}_{1}^{T} \mathscr{R} \hat{\chi},
\end{array}\right. \\
& \left\{\begin{array}{l}
(\beta-\alpha) \int_{\alpha}^{\beta} \dot{e}^{T}(s) \mathscr{R} \dot{e}(s) \mathrm{d} s, \quad \geq \chi_{1}^{T} \mathscr{R} \chi_{1}+3 \chi_{2}^{T} \mathscr{R} \chi_{2}, \\
(\beta-\alpha) \int_{\alpha}^{\beta} e^{T}(s) \mathscr{R} e(s) \mathrm{d} s, \quad \geq \hat{\chi}_{1}^{T} \mathscr{R} \hat{\chi}_{2}+3 \hat{\chi}_{2}^{T} \mathscr{R} \hat{\chi}_{2},
\end{array}\right. \\
& (\beta-\alpha) \int_{\alpha}^{\beta} \dot{e}^{T}(s) \mathscr{R} \dot{e}(s) \mathrm{d} s \geq \chi_{1}^{T} \mathscr{R} \chi_{1}+3 \chi_{2}^{T} \mathscr{R} \chi_{2}+5 \chi_{3}^{T} \mathscr{R} \chi_{3},
\end{aligned}
$$

where $\chi_{1}=e(\beta)-e(\alpha), \chi_{2}=e(\beta)+e(\alpha)-(2 /(\beta-\alpha)) \int_{\alpha}^{\beta} e$ $(s) d s, \quad \hat{\chi}_{1}=\int_{\alpha}^{\beta} e(s) d s, \hat{\chi}_{2}=\int_{\alpha}^{\beta} e(s) d s-(2 /(\beta-\alpha)) \int_{\alpha}^{\beta} \int_{\theta}^{\beta} e$ (s) $d s d \theta$, and $\chi_{3}=e(\beta)-x(\alpha)+(6 /(\beta-\alpha)) \int_{\alpha}^{\beta} e(s) d s-$ $\left(12 /(\beta-\alpha)^{2}\right) \int_{\alpha}^{\beta} \int_{\theta}^{\beta} e(s) d s d \theta$.

\section{Main Results}

In this section, we will provide a novel RMFC design scheme for (12). In the following theorem, the asymptotic condition for system (13) is provided under the designed gain matrices $\mathscr{K}_{i}(i=1,2,3)$. For simplicity, some relevant notations are defined as in Appendix A.
Theorem 1. Given positive scalars $h, \mu$, and $\varepsilon$. System (13) is asymptotically stable if there exist symmetric positive definite matrices $\mathscr{P}_{1}, \mathscr{M}_{2}, \mathscr{M}_{3}, \mathscr{G}_{1}, \mathscr{G}_{2}, \mathscr{D}_{1}$, and $\mathscr{D}_{2}$, any symmetric matrices $\mathscr{P}_{2}, \mathscr{M}_{1}, \mathscr{M}_{4}$, and $\mathbb{Q}_{i}(i=1,2, \ldots, 5)$, any matrices $\mathcal{U}_{1}, \mathcal{U}_{2}, \mathcal{N}_{1}$ and $\mathcal{N}_{2}$ with appropriate dimensions such that the following LMIs hold:

$$
\begin{aligned}
& \mathscr{P}_{h(t)=0}>0, \\
& \mathscr{M}_{1, h(t)=h}>0 \text {, } \\
& \mathscr{M}_{2, h(t)=0}>0 \text {, } \\
& {\left[\begin{array}{cccc}
\Sigma_{1} & \frac{\sqrt{h}}{h} \mathscr{E}_{1}^{T} \mathcal{U}_{2} & \Gamma & \Theta \\
* & -\Pi_{2, \dot{h}(t)} & 0 & 0 \\
* & * & -\varepsilon^{-1} \mathscr{I} & 0 \\
* & * & * & -\varepsilon \mathscr{I}
\end{array}\right]<0,} \\
& {\left[\begin{array}{cccc}
\Sigma_{2} & \frac{\sqrt{h}}{h} \mathscr{E}_{1}^{T} \mathcal{U}_{2} & \Gamma & \Theta \\
* & -\Pi_{2, \dot{h}(t)} & 0 & 0 \\
* & * & -\varepsilon^{-1} \mathscr{I} & 0 \\
* & * & * & -\varepsilon \mathscr{I}
\end{array}\right]<0,} \\
& {\left[\begin{array}{cccc}
\Sigma_{3} & \frac{\sqrt{h}}{h} \mathscr{E}_{2}^{T} \mathscr{U}_{1} & \Gamma & \Theta \\
* & -\Pi_{1, \dot{h}(t)} & 0 & 0 \\
* & * & -\varepsilon^{-1} \mathscr{I} & 0 \\
* & * & * & -\varepsilon \mathscr{I}
\end{array}\right]<0,} \\
& {\left[\begin{array}{cccc}
\Sigma_{4} & \frac{\sqrt{h}}{h} \mathscr{E}_{2}^{T} \mathscr{U}_{1} & \Gamma & \Theta \\
* & -\Pi_{1, \dot{h}(t)} & 0 & 0 \\
* & * & -\varepsilon^{-1} \mathscr{I} & 0 \\
* & * & * & -\mathcal{E} \mathscr{I}
\end{array}\right]<0,}
\end{aligned}
$$

where other symbols and related equations are listed in Appendix B.

Proof. Construct an augmented LKF as follows:

$$
V(x(t))=\sum_{i=1}^{5} V_{i}(x(t)),
$$

where 


$$
\begin{aligned}
& V_{1}(x(t))=\alpha^{T}(t) \mathscr{P}_{h(t)} \alpha(t), \\
& V_{2}(x(t))=\eta^{T}(t) \mathscr{Q} \eta(t), \\
& V_{3}(x(t))=\int_{t-h(t)}^{t} \dot{x}^{T}(s) \mathscr{M}_{1, h(t)} \dot{x}(s) \mathrm{d} s+\int_{t-h}^{t-h(t)} \dot{x}^{T}(s) \mathscr{M}_{2, h(t)} \dot{x}(x) \mathrm{d} s, \\
& V_{4}(x(t))=\int_{-h(t)}^{0} \int_{t+\theta}^{t} \dot{x}^{T}(s) \mathscr{G}_{1} \dot{x}(s) \mathrm{d} s \mathrm{~d} \theta+\int_{-h}^{-h(t)} \int_{t+\theta}^{t} \dot{x}^{T}(s) \mathscr{G}_{2} \dot{x}(s) \mathrm{d} s \mathrm{~d} \theta, \\
& V_{5}(x(t))=2 \sum_{i=1}^{n} d_{1 i} \int_{0}^{x(t-h(t))}\left(f_{i}(s)-l_{i}^{-} s\right) \mathrm{d} s+2 \sum_{i=1}^{n} d_{2 i} \int_{0}^{x(t-h(t))}\left(l_{i}^{+} s-f_{i}(s)\right) \mathrm{d} s,
\end{aligned}
$$

$\alpha(t), \eta(t), \mathscr{P}_{h(t)}, \mathcal{Q}, \mathscr{M}_{1, h(t)}, \mathscr{M}_{2, h(t)}$, and $\xi(t)$ are given in Appendix A.
The time derivative of $V(x(t))$ along the trajectory of system (12) is given. Then, the derivative of $V_{i}(x(t))$ is derived:

$$
\begin{aligned}
& \dot{V}_{1}(x(t))=2 \alpha^{T}(t) \mathscr{P}_{h(t)} \dot{\alpha}(t)+\dot{h}(t) \alpha^{T}(t) \mathscr{P}_{2} \alpha(t) \\
& =\dot{h}(t) \alpha^{T}(t) \mathscr{P}_{2} \alpha(t)+2 \alpha^{T}(t) \mathscr{P}_{h(t)} \\
& \times\left[\begin{array}{c}
\dot{x}(t) \\
\dot{x}(t-h) \\
x(t)-(1-\dot{h}(t)) x(t-h(t)) \\
(1-\dot{h}(t)) x(t-h(t))-x(t-h)
\end{array}\right] \\
& =\xi^{T}(t)\left\{\dot{h}(t) \Phi_{1}^{T}(h(t)) \mathscr{P}_{2} \Phi_{1}(h(t))+\operatorname{Sym}\left\{\Phi_{1}^{T}(h(t)) \mathscr{P}_{h(t)} \Phi_{2}(\dot{h}(t))\right\}\right\} \xi(t), \\
& \dot{V}_{2}(x(t))=2 \eta^{T}(t)\left[\begin{array}{ccc}
\frac{Q_{1}^{T}+Q_{1}-Q_{2}^{T}-Q_{2}}{2} & Q_{2} & Q_{3} \\
* & \frac{-Q_{1}^{T}-Q_{1}-Q_{2}^{T}-Q_{2}}{2} & Q_{4} \\
* & * & Q_{5}^{T}+Q_{5}
\end{array}\right] \dot{\eta}(t) \\
& =2 \eta^{T}(t)\left[\begin{array}{ccc}
\frac{Q_{1}^{T}+Q_{1}-Q_{2}^{T}-Q_{2}}{2} & Q_{2} & Q_{3} \\
* & \frac{-Q_{1}^{T}-Q_{1}-Q_{2}^{T}-Q_{2}}{2} & Q_{4} \\
* & * & Q_{5}^{T}+Q_{5}
\end{array}\right]\left[\begin{array}{c}
\dot{x}(t) \\
(1-\dot{h}(t)) \dot{x}(t-h(t)) \\
\dot{x}(t-h)
\end{array}\right] \\
& =\xi^{T}(t) \operatorname{Sym}\left\{\Phi_{3}^{T} Q \Phi_{4}(\dot{h}(t))\right\} \xi(t) \\
& \dot{V}_{3}(x(t))=\dot{x}^{T}(t) \mathscr{M}_{1, h(t)} \dot{x}(t)-(1-\dot{h}(t)) \dot{x}(t-h(t)) \mathscr{M}_{1, h(t)} \dot{x}(t-h(t))+(1-\dot{h}(t)) \\
& \times \dot{x}^{T}(t-h(t)) \mathscr{M}_{2, h(t)} \dot{x}(t-h(t))-\dot{x}(t-h) \mathscr{M}_{2, h(t)} \dot{x}(t-h) \\
& +\dot{h}(t) \int_{t}^{t-h(t)} \dot{x}^{T}(s) \mathscr{M}_{1} \dot{x}(s) \mathrm{d} s-\dot{h}(t) \int_{t-h(t)}^{t-h} \dot{x}^{T}(s) \mathscr{M}_{1} \dot{x}(s) \mathrm{d} s,
\end{aligned}
$$




$$
\begin{aligned}
\dot{V}_{4}(x(t))= & h(t) \dot{x}^{T}(t) \mathscr{G}_{1} \dot{x}(t)+(h-h(t)) \dot{x}^{T}(t) \mathscr{G}_{2} \dot{x}(t)-(1-\dot{h}(t)) \int_{t-h(t)}^{t} \dot{x}^{T}(s) \mathscr{G}_{1} \dot{x}(s) \mathrm{d} s \\
& -\dot{h}(t) \int_{t-h(t)}^{t} \dot{x}^{T}(s) \mathscr{G}_{2} \dot{x}(s) \mathrm{d} s-\int_{t-h}^{t-h(t)} \dot{x}^{T}(s) \mathscr{G}_{2} \dot{x}(s) \mathrm{d} s .
\end{aligned}
$$

Combining (24) and (25), we can get the derivative of $\dot{V}_{T}(x(t))$ as follows:

$$
\begin{aligned}
V_{T}(x(t))= & \dot{x}^{T}(t)\left[\mathscr{G}_{h(t)}+\mathscr{M}_{1, h(t)}\right] \dot{x}(t)+(1-\dot{h}(t)) \dot{x}^{T}(t-h(t))\left[\mathscr{M}_{2, h(t)}-\mathscr{M}_{1, h(t)}\right] \dot{x}(t-h(t)) \\
& -\dot{x}^{T}(t-h) \mathscr{M}_{2, h(t)} \dot{x}(t-h)-\int_{t-h(t)}^{t} \dot{x}^{T}(s) \bar{\Pi}_{1, \dot{h}(t)} \dot{x}(s) \mathrm{d} s-\int_{t-h}^{t-h(t)} \dot{x}^{T}(s) \bar{\Pi}_{2, \dot{h}(t)} \dot{x}(s) \mathrm{d} s .
\end{aligned}
$$

Based on Lemma 1, we can get

$$
\begin{aligned}
& -\int_{t-h(t)}^{t} \dot{x}^{T}(s) \bar{\Pi}_{1, \dot{h}(t)} \dot{x}(s) \mathrm{d} s-\int_{t-h}^{t-h(t)} \dot{x}^{T}(s) \bar{\Pi}_{2, \dot{h}(t)} \dot{x}(s) \mathrm{d} s \\
& \leq \xi^{T}(t)\left(\frac{h-h(t)}{h^{2}} \mathscr{E}_{1}^{T} \mathcal{U}_{2}^{T} \Pi_{2, \dot{h}(t)}^{-1} \mathcal{U}_{2} \mathscr{E}_{1}+\frac{h(t)}{h^{2}} \mathscr{E}_{2}^{T} \mathcal{U}_{1}^{T} \Pi_{1, \dot{h}(t)}^{-1}\right. \\
& \left.\times \mathcal{S}_{3} \mathscr{W}_{2}-\frac{1}{h}\left[\begin{array}{c}
\mathscr{E}_{1} \\
\mathscr{E}_{2}
\end{array}\right]^{T}\left[\begin{array}{cc}
\frac{2 h-h(t)}{h} \bar{\Pi}_{1, \dot{h}(t)} & \frac{h-h(t)}{h} \mathcal{U}_{1}+\frac{h(t)}{h} \mathcal{U}_{2} \\
* & \frac{1}{h-h(t)} \bar{\Pi}_{2, \dot{h}(t)}
\end{array}\right]\left[\begin{array}{l}
\mathscr{E}_{1} \\
\mathscr{E}_{2}
\end{array}\right]\right) \xi(t) \\
& =\xi^{T}(t)\left\{\Psi_{h(t)}-\frac{1}{h}\left(\frac{2 h-h(t)}{h} \mathscr{E}_{1}^{T} \Pi_{1, \dot{h}(t)} \mathrm{E}_{1}+\frac{h-h(t)}{h} \mathscr{E}_{2}^{T} \mathcal{U}_{1}^{T} \mathscr{E}_{1}+\frac{h-h(t)}{h} \mathscr{E}_{2}^{T}\right.\right. \\
& \left.\left.\times \mathscr{U}_{2}^{T} \mathscr{E}_{1}+\frac{h-h(t)}{h} \mathscr{E}_{1}^{T} \mathcal{U}_{1} \mathscr{E}_{2}+\frac{h-h(t)}{h} \mathscr{E}_{1}^{T} \mathscr{U}_{2} \mathscr{E}_{2}+\frac{h-h(t)}{h} \mathscr{E}_{2}^{T} \Pi_{2, \dot{h}(t)} \mathscr{E}_{2}\right)\right\} \xi(t) \\
& =\xi^{T}(t)(\Psi(h(t))+\Omega(h(t))) \xi(t) .
\end{aligned}
$$

Then, $\dot{V}_{T}(x(t))$ can be expressed as follows:

$$
\begin{gathered}
\dot{V}_{T}(x(t)) \leq \xi^{T}(t)\left(e_{4}^{T}\left[\mathscr{G}_{h(t)}+\mathscr{M}_{1, h(t)}\right] e_{4}+(1-\dot{h}(t)) e_{5}^{T}\left[\mathscr{M}_{2, h(t)}-\mathscr{M}_{1, h(t)}\right] e_{5}\right. \\
\left.-e_{6}^{T} \mathscr{M}_{2, h(t)} e_{6}+\Psi(h(t))+\Omega(h(t))\right) \xi(t),
\end{gathered}
$$

where $\mathscr{G}_{h(t)}, \Pi_{1, \dot{h}(t)}, \Pi_{2, \dot{h}(t)}, \Psi(h(t))$, and $\Omega(h(t))$ are given in Appendix B.

$$
\begin{aligned}
\dot{V}_{5}(x(t))= & 2(1-\dot{h}(t)) f^{T}(x(t-h(t)))\left(\mathscr{D}_{1}-\mathscr{D}_{2}\right) \dot{x}(t-h(t)) \\
& +2(1-\dot{h}(t)) x^{T}(t-h(t))\left(\mathscr{D}_{2} \mathscr{L}_{2}-\mathscr{D}_{1} \mathscr{L}_{1}\right) \dot{x}(t-h(t)) \\
= & \xi^{T}(t) \Xi_{4} \xi(t) .
\end{aligned}
$$


Based on system (13), the following zero formula holds:

$$
\begin{aligned}
0 & =2\left[x^{T}(t) \mathscr{N}_{1}+\dot{x}(t) \mathscr{N}_{2}\right]\left[\begin{array}{c}
\left(-\mathscr{A}+\Delta \mathscr{A}(t)+(\mathscr{I}-\Pi) \mathscr{K}_{1}\right) x(t)+\mathscr{W}_{0} f(x(t)) \\
+\mathscr{W}_{1} f(x(t-h(t)))+(\mathscr{I}-\Pi) \mathscr{K}_{2} x(t-h(t))+(\mathscr{I}-\Pi) \mathscr{K}_{3} x(t-h)-\dot{x}(t)
\end{array}\right] \\
& =\xi^{T}(t)\left(\operatorname{Sym}\{\mathscr{L} \varphi\}+2 \mathscr{L} \Delta \mathscr{A}(t) e_{1}\right) \xi(t) .
\end{aligned}
$$

Then, based on Lemma 3 in [34], we can get

$$
\begin{aligned}
2 \mathscr{L} \Delta \mathscr{A}(t) e_{1} \leq \varepsilon(\mathscr{L} \mathscr{H})(\mathscr{L} \mathscr{H})^{T} & +\varepsilon^{-1}\left(\mathscr{E} e_{1}\right)\left(\mathscr{E} e_{1}\right)^{T} \\
& =\varepsilon \Gamma \Gamma^{T}+\varepsilon^{-1} \Theta \Theta^{T} .
\end{aligned}
$$

Based on the convex combination technique, $\sum_{i=1}^{5} \dot{V}(x(t))<0$ holds for all, if only if. By utilizing Schur complement, we can derive that $\sum_{i=1}^{5} \dot{V}(x(t))<0$ is equal to

$$
\left[\begin{array}{ccccc}
\Sigma_{h(t), \dot{h}(t)} & \frac{\sqrt{h-h(t)}}{h} \mathscr{E}_{1}^{T} \mathcal{U}_{2} & \frac{\sqrt{h(t)}}{h} \mathscr{E}_{2}^{T} \mathcal{U}_{1} & \Gamma & \Theta \\
* & -\Pi_{2, \dot{h}(t)} & 0 & 0 & 0 \\
* & * & -\Pi_{1, \dot{h}(t)} & 0 & 0 \\
* & * & * & -\mathcal{E}^{-1} \mathscr{J} & 0 \\
* & * & * & * & -\mathcal{E} \mathscr{J}
\end{array}\right]<0 .
$$

Based on (15)-(19), it is easy to come to the conclusion that system (13) is asymptotically stable. This concludes the proof.

Remark 2. In this paper, we consider $\mathscr{P}_{h(t)}=\mathscr{P}_{1}-(h-h(t)) \mathscr{P}_{2}$. (I) When $h-h(t)=0, \mathscr{P}_{h(t)}$ will be degenerated to the constant matrix $\mathscr{P}$. (II) Compared with the existing methods [25], this paper only needs to consider that $\mathscr{P}_{2}$ and $\mathscr{P}_{2}$ are arbitrary symmetric matrices. Furthermore, as long as (25) and (26) are guaranteed, this constraint helps reduce the strength of positive definite conditions. (III) In addition, this construction method makes full use of the delay information and the delay derivative information, thereby increasing the amount of LKF information storage, which helps to construct a more general LKF to further reduce the conservativeness of the criteria. At present, the method used in this paper is more general in constructing the LKF and includes a wider range of usage background and research significance.

Remark 3. Compared with existing research [24], this paper fully considers the relaxation of the requirements for matrix positive definiteness. In $V_{3}(x(t))$, by using $\mathscr{M}_{1, h(t)}$ and $\mathscr{M}_{2, h(t)}$ to replace the constant matrices, the LCCM is used to make constraints. Therefore, this method can obtain less conservative criteria through more relaxed positive definite conditions and increase the time-delay information contained in the LKF.

Remark 4. In order to better solve integral terms (24) and $(25),-\int_{t-h(t)}^{t} \dot{x}^{T}(s) \bar{\Pi}_{1, \dot{h}(t)} \dot{x}(s) \mathrm{d} s-\int_{t-h}^{t-h(t)} \dot{x}^{T}(s) \bar{\Pi}_{2, \dot{h}(t)} \dot{x}(s) \mathrm{d} s$, in this paper, considers Lemma 1 . Compared with the Wirtinger-based integral inequality, Jensen's inequality, and other existing inequalities, Lemma 1 has a tighter bound in order to obtain a less conservative criterion.

Theorem 2. Given positive scalars $h, \mu, \varepsilon$, and $\kappa$. System (13) is asymptotically stable if there exist symmetric positive definite matrices $\widetilde{\mathscr{P}}_{1}, \widetilde{\mathscr{M}}_{2}, \widetilde{\mathscr{M}}_{3}, \widetilde{\mathscr{G}}_{1}, \widetilde{\mathscr{G}}_{2}, \widetilde{\mathscr{D}}_{1}$, and $\widetilde{\mathscr{D}}_{2}$. Any symmetric matrices $\widetilde{\mathscr{P}}_{2}, \widetilde{\mathscr{M}}_{1}, \widetilde{\mathscr{M}}_{4}$, and $\widetilde{Q}_{i}(i=1,2, \ldots, 5)$, any matrices $\widetilde{\mathscr{U}}_{1}, \widetilde{\mathcal{U}}_{2}, \widetilde{\mathcal{Y}}_{1}, \widetilde{\mathscr{Y}}_{2}, \widetilde{\mathscr{Y}}_{3}$ and $\tilde{\mathscr{V}}_{\text {with appropriate }}$ dimensions such that the following LMIs hold:

$$
\begin{aligned}
& \widetilde{\mathscr{P}}_{h(t)=0}>0, \\
& \widetilde{\mathscr{M}}_{1, h(t)=h}>0 \text {, } \\
& \widetilde{\mathscr{M}}_{2, h(t)=0}>0 \text {, } \\
& {\left[\begin{array}{cccc}
\widetilde{\Sigma}_{1} & \frac{\sqrt{h}}{h} \mathscr{E}_{1}^{T} \widetilde{\mathscr{U}}_{2} & \widetilde{\Gamma} & \widetilde{\Theta} \\
* & -\widetilde{\Pi}_{2, \dot{h}(t)} & 0 & 0 \\
* & * & -\varepsilon^{-1} \mathscr{I} & 0 \\
* & * & * & -\varepsilon \mathscr{I}
\end{array}\right]<0,} \\
& {\left[\begin{array}{cccc}
\widetilde{\Sigma}_{2} & \frac{\sqrt{h}}{h} \mathscr{E}_{1}^{T} \widetilde{\mathscr{U}}_{2} & \widetilde{\Gamma} & \widetilde{\Theta} \\
* & -\widetilde{\Pi}_{2, \dot{h}(t)} & 0 & 0 \\
* & * & -\varepsilon^{-1} \mathscr{I} & 0 \\
* & * & * & -\varepsilon \mathscr{I}
\end{array}\right]<0,} \\
& {\left[\begin{array}{cccc}
\widetilde{\Sigma}_{3} & \frac{\sqrt{h}}{h} \mathscr{E}_{2}^{T} \widetilde{\mathscr{U}}_{1} & \widetilde{\Gamma} & \widetilde{\Theta} \\
* & -\widetilde{\Pi}_{1, \dot{h}(t)} & 0 & 0 \\
* & * & -\varepsilon^{-1} \mathscr{I} & 0 \\
* & * & * & -\varepsilon \mathscr{I}
\end{array}\right]<0,}
\end{aligned}
$$




$$
\left[\begin{array}{cccc}
\widetilde{\Sigma}_{4} & \frac{\sqrt{h}}{h} \mathscr{E}_{2}^{T} \widetilde{U}_{1} & \widetilde{\Gamma} & \widetilde{\Theta} \\
* & -\widetilde{\Pi}_{1, \dot{h}(t)} & 0 & 0 \\
* & * & -\varepsilon^{-1} \mathscr{I} & 0 \\
* & * & * & -\mathcal{E} \mathscr{I}
\end{array}\right]<0,
$$

where other symbols and related equations are listed in Appendix $C$.

We define $\quad \mathscr{K}_{1}=\mathscr{Y}_{1} \mathscr{V}^{-1}, \quad \mathscr{K}_{2}=\mathscr{Y}_{2} \mathscr{V}^{-1}, \quad$ and $\mathscr{K}_{3}=\mathscr{Y}_{3} \mathscr{V}^{-1}$ to solve the corresponding controller gain.

Proof. Define

$$
\begin{aligned}
& \mathcal{N}_{1}=\mathscr{V}^{-1}, \\
& \mathcal{N}_{2}=\kappa \mathscr{V}^{-1} \text {, } \\
& \widetilde{\mathscr{P}}_{h(t)}=\operatorname{diag}\left\{\mathscr{V}^{T}, \mathscr{V}^{T}, \mathscr{V}^{T}, \mathscr{V}^{T}\right\} \mathscr{P}_{h(t)} \operatorname{diag}\{\mathscr{V}, \mathscr{V}, \mathscr{V}, \mathscr{V},\}, \\
& \widetilde{\mathscr{G}}_{1}=\mathscr{V}^{T} \mathscr{G}_{1} \mathscr{V} \\
& \widetilde{\mathscr{G}}_{2}=\mathscr{V}^{T} \mathscr{G}_{2} \mathscr{V} \\
& \widetilde{Q}_{i}=\mathscr{V}^{T} \mathscr{P}_{h(t)} \mathscr{V}, \\
& \widetilde{\mathscr{M}}_{1}=\mathscr{V}^{T} \mathscr{M}_{1} \mathscr{V} \text {, } \\
& \widetilde{\mathscr{M}}_{2}=\mathscr{V}^{T} \mathscr{M}_{2} \mathscr{V} \text {, } \\
& \widetilde{\mathscr{M}}_{3}=\mathscr{V}^{T} \mathscr{M}_{3} \mathscr{V} \text {, } \\
& \widetilde{\mathscr{M}}_{4}=\mathscr{V}^{T} \mathscr{M}_{4} \mathscr{V} \text {, } \\
& \widetilde{\mathscr{U}}_{1}=\operatorname{diag}\left\{\mathscr{V}^{T}, \mathscr{V}^{T}, \mathscr{V}^{T}\right\} \mathscr{U}_{1} \operatorname{diag}\{\mathscr{V}, \mathscr{V}, \mathscr{V}\}, \\
& \tilde{\mathscr{D}}_{1}=\mathscr{V}^{T} \mathscr{M}_{1} \mathscr{V} \text {, } \\
& \widetilde{\mathscr{U}}_{2}=\operatorname{diag}\left\{\mathscr{V}^{T}, \mathscr{V}^{T}, \mathscr{V}^{T}\right\} \mathcal{U}_{2} \operatorname{diag}\{\mathscr{V}, \mathscr{V}, \mathscr{V}\}, \\
& \tilde{\mathscr{D}}_{2}=\mathscr{V}^{T} \mathscr{M}_{2} \mathscr{V} \text {. }
\end{aligned}
$$

Premultiplying and postmultiplying (15)-(19) by $\operatorname{diag}\left\{\mathscr{V}^{T}, \mathscr{V}^{T}, \mathscr{V}^{T}, \mathscr{V}^{T}\right\}$ and $\operatorname{diag}\{\mathscr{V}, \mathscr{V}, \mathscr{V}, \mathscr{V}\}$, in view of the Schur complement, we can obtain LMIs (33)-(37). Thus, the proof is completed.

\section{Illustrative Example}

In this section, two simulation examples are exhibited to express the effectiveness of the established results.
Example 1. Consider DNN (13) with the following matrix parameters, and these matrices are based on [13-18]:

$$
\begin{aligned}
\mathscr{A} & =\left[\begin{array}{ll}
2 & 0 \\
0 & 2
\end{array}\right], \\
\mathscr{W}_{0} & =\left[\begin{array}{cc}
1 & 1 \\
-1 & -1
\end{array}\right], \\
\mathscr{W}_{1} & =\left[\begin{array}{ll}
0.88 & 1 \\
1 & 1
\end{array}\right], \\
\mathscr{L}_{1} & =\operatorname{diag}\{0.4,0.8\}, \\
\mathscr{L}_{2} & =0, \\
\Pi & =\operatorname{diag}\{0.5,0.5,0.5,0.5\} .
\end{aligned}
$$

The MAUBs are obtained by Theorem 1, and the other results are listed in Table 1 . When $\mu_{2}=0.8$, from Table 2, we can clearly see that our result is markedly better than $[13-18,25]$. In addition, it is worth noting that the result obtained in this paper is improved by $21.8325 \%$ compared to [25]. For the reason that the TDPF method is employed, the conservativeness of the obtained criterion is reduced and the performance is improved in this paper.

Setting $\mu=1, h(t) \leq 6.0917, h(t)=(60917 / 20000) \sin$ $((20000 / 60917) t)+(60917 / 20000)$, and $f(x(t))=\operatorname{diag}$ $(0.4,0.8) \tanh (x(t))$. In order to verify the stability of the system, we randomly select different initial values to simulate the dynamic response of the system as shown in Figure 1. From Figure 1, we can clearly see that when different initial values are selected, the system tends to a stable state as time increases.

Based on Theorem 2, setting $\kappa=1$, we can get the controller gains as follows:

$$
\begin{aligned}
& \mathscr{K}_{1}=\left[\begin{array}{cc}
-0.1488 & -0.4091 \\
0.2762 & -0.2079
\end{array}\right], \\
& \mathscr{K}_{2}=\left[\begin{array}{cc}
-0.2986 & 0.1950 \\
-0.7407 & -0.1002
\end{array}\right], \\
& \mathscr{K}_{3}=\left[\begin{array}{cc}
-0.5131 & -0.3584 \\
0.2236 & -0.1432
\end{array}\right] .
\end{aligned}
$$

Here, the control input trajectory of DNNs is presented in Figure 2. 
TABLE 1: Notations and descriptions.

\begin{tabular}{|c|c|c|c|}
\hline Notation & $\mathbb{R}^{m}$ & $\mathbb{R}^{l \times k}$ & $\mathscr{I}$ \\
\hline Description & $m$-dimensional Euclidean space & $l \times k$ matrices & Identity matrix \\
\hline Notation & $\mathscr{P}>0$ & $\mathscr{A}^{-1}$ & $\mathscr{A}^{T}$ \\
\hline Description & Positive definite matrix & Inverse of matrix $\mathscr{A}$ & Inverse of matrix $\mathscr{A}$ \\
\hline Notation & $\|\cdot\|$ & $\operatorname{sym}\{X\}$ & $\operatorname{diag}\{\cdots\}$ \\
\hline Description & Inverse of matrix $\mathscr{A}$ & $X^{T}+X$ & Stands for a block diagonal matrix \\
\hline
\end{tabular}

TABle 2: The achieved MAUBs for different values of $\mu$.

\begin{tabular}{lccc}
\hline Methods & $\mu=0.1$ & $\mu=0.5$ & $\mu=0.9$ \\
\hline$[19]$ & 3.3068 & 2.5802 & 2.2736 \\
{$[22]$} & 3.8025 & 2.7427 & 2.3811 \\
{$[23]$} & 3.8026 & 2.7440 & 2.3811 \\
{$[25]$} & 4.6754 & 3.0587 & 2.7368 \\
Theorem 1 & 5.5721 & 4.0916 & 3.9594 \\
Improvement & $16.0927 \%$ & $25.2444 \%$ & $30.8784 \%$ \\
\hline
\end{tabular}

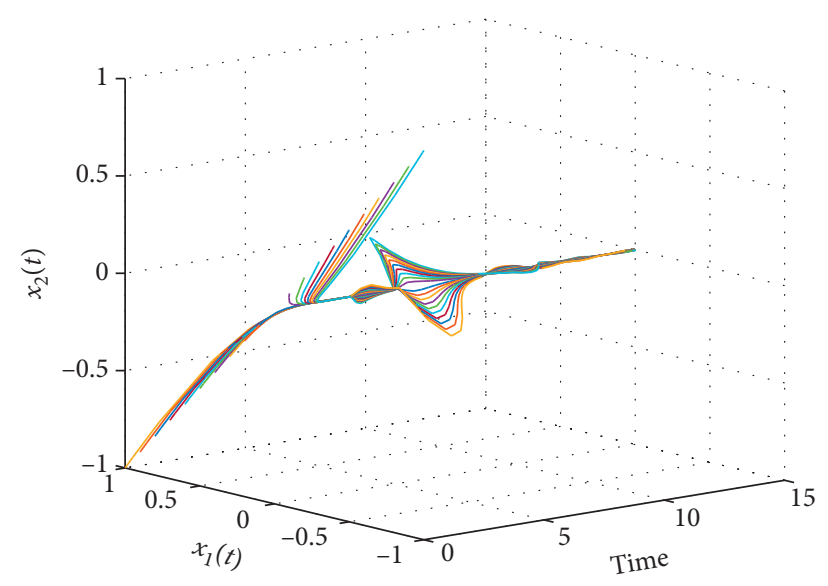

FIGURE 1: State trajectories for $\mu=1$ and $h=6.0917$.

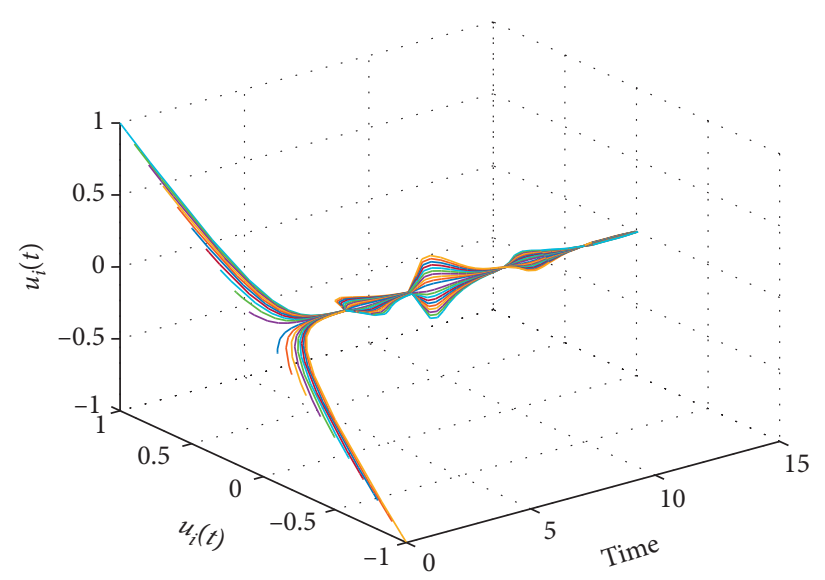

FIgUre 2: Control input of the system. 
TABle 3: The achieved MAUBs for different values of $\mu$.

\begin{tabular}{lcr}
\hline Methods & $\mu=0.8$ & $\mu=0.9$ \\
\hline$[13]$ & 2.5406 & 1.7273 \\
{$[14]$} & 2.2495 & 1.5966 \\
{$[15]$} & 2.1105 & 1.4268 \\
{$[16]$} & 2.8794 & 1.9562 \\
{$[17]$} & 2.8980 & 1.9562 \\
{$[15]$} & 3.1409 & 1.6375 \\
{$[18]$} & 4.8167 & 3.4245 \\
{$[25]$} & 5.2756 & 4.6281 \\
Theorem 1 & 6.7491 & 5.9291 \\
Improvement & $21.8325 \%$ & $21.9426 \%$ \\
\hline
\end{tabular}

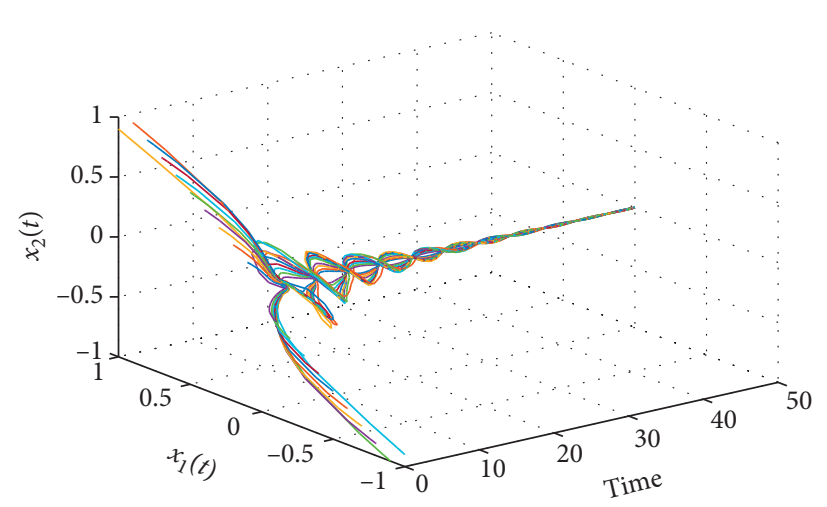

Figure 3: State trajectories $x_{1}(t)$ and $x_{2}(t)$ for $\mu=0.1$ and $h=4.2290$.

Example 2. Consider DNN (13) with the following matrix parameters, and these matrices are based on [19-25]:

$$
\begin{aligned}
\mathscr{A} & =\operatorname{diag}\{1.2769,0.6231,0.9230,0.4480\}, \\
\mathscr{L}_{1} & =\operatorname{diag}\{0.1137,0.1297,0.7994,0.2368\}, \\
\mathscr{W}_{0} & =\left[\begin{array}{cccc}
-0.0373 & 0.4852 & -0.3351 & 0.2336 \\
-1.6033 & 0.5988 & 0.3224 & 1.2352 \\
0.3394 & -0.0860 & -0.3824 & -0.5785 \\
-0.1311 & 0.3253 & -0.9534 & -0.5015
\end{array}\right], \\
\mathscr{W}_{1} & =\left[\begin{array}{cccc}
0.8674 & -1.2405 & -0.5325 & 0.0220 \\
0.0474 & -0.9164 & 0.0360 & 0.9816 \\
1.8495 & 2.6117 & -0.3788 & 0.8428 \\
-2.0413 & 0.5179 & 1.1734 & -0.2775
\end{array}\right], \\
\mathscr{L}_{2} & =0, \\
\Pi & =\operatorname{diag}\{0.5,0.5,0.5,0.5\} .
\end{aligned}
$$

By employing Theorem 1, for different $\mu$, Table 3 lists the MAUBs based on Theorem 1. From Table 3, we can see that when $\mu$ takes different values, the MAUBs obtained in this paper are always the largest. For example, when $\mu=0.5$, the existing result shows $h=3.0587$, but the result obtained by applying Theorem 1 in this paper is $h=4.0916$. Compared with results in [25], the criterion proposed in this paper improves MAUBs by up to $25.2444 \%$.
When $\kappa=1$, by solving LMIs in Theorem 2, the controller gains are obtained as follows:

$$
\begin{aligned}
& \mathscr{K}_{1}=\left[\begin{array}{llll}
-2.8807 & -1.3407 & -0.4210 & -0.1567 \\
-0.1579 & -0.1483 & -0.1396 & 1.5440 \\
-1.9637 & -1.0886 & -0.1666 & -0.1519 \\
-0.1573 & -0.1539 & 0.9762 & 1.9872
\end{array}\right], \\
& \mathscr{K}_{2}=\left[\begin{array}{cccc}
-1.6769 & -0.1564 & -0.1578 & -0.1525 \\
-0.1651 & 0.3745 & 1.3234 & 3.1923 \\
-0.1484 & -0.1550 & -0.1652 & -0.1642 \\
-0.1488 & -0.4091 & 0.2762 & -0.2079
\end{array}\right], \\
& \mathscr{K}_{3}=\left[\begin{array}{cccc}
-0.1979 & -0.1569 & -0.1676 & -0.2986 \\
0.1950 & -0.7407 & -0.1002 & -0.2256 \\
-0.1683 & -0.1543 & -0.5131 & -0.3584 \\
-0.1432 & 0.3253 & -0.1054 & -0.2237
\end{array}\right] .
\end{aligned}
$$

Letting $\mu=0.1, h(t) \leq 4.2290, f(x(t))=\operatorname{diag}(0.1137$, $0.1297,0.7994,0.2368) \tanh (x(t))$, and $h(t)=(42290 / 10000)$ $\sin ((1000 / 42290) t)+(42290 / 10000)$. We verify the stability of the system by choosing different initial values. As shown in Figures 3-8, we give a dynamic response graph through node combination. Through the figures, we can directly see that the system eventually tends to a stable state under the controller involved. In addition, the control input trajectory of DNNs is presented in Figure 9. 


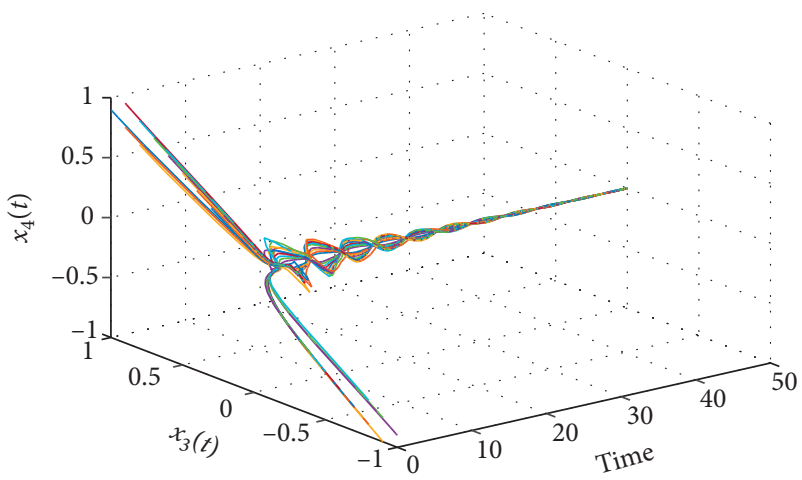

Figure 4: State trajectories $x_{3}(t)$ and $x_{4}(t)$ for $\mu=0.1$ and $h=4.2290$.

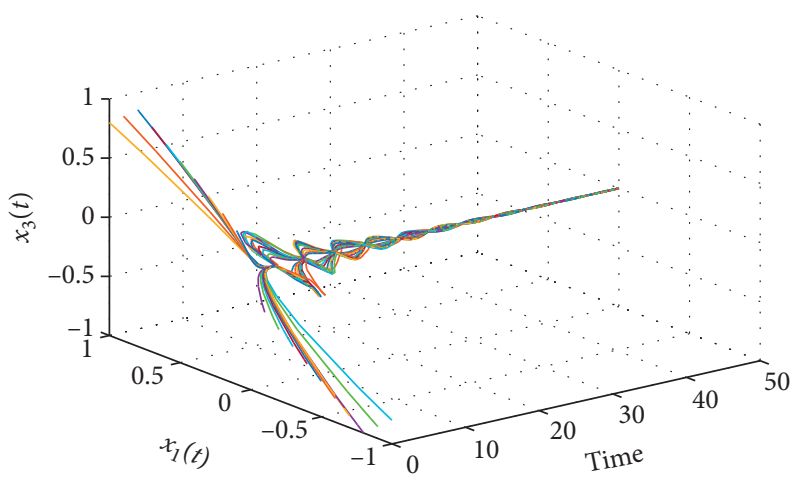

Figure 5: State trajectories $x_{1}(t)$ and $x_{3}(t)$ for $\mu=0.1$ and $h=4.2290$.

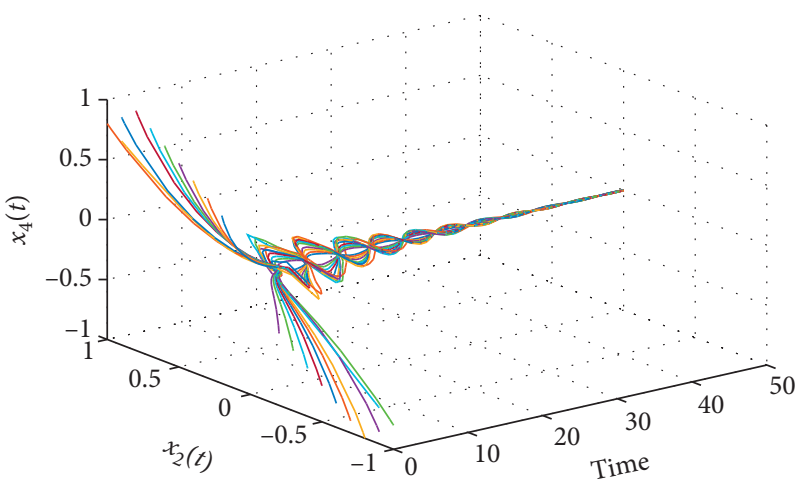

FIGURE 6: State trajectories $x_{2}(t)$ and $x_{4}(t)$ for $\mu=0.1$ and $h=4.2290$. 


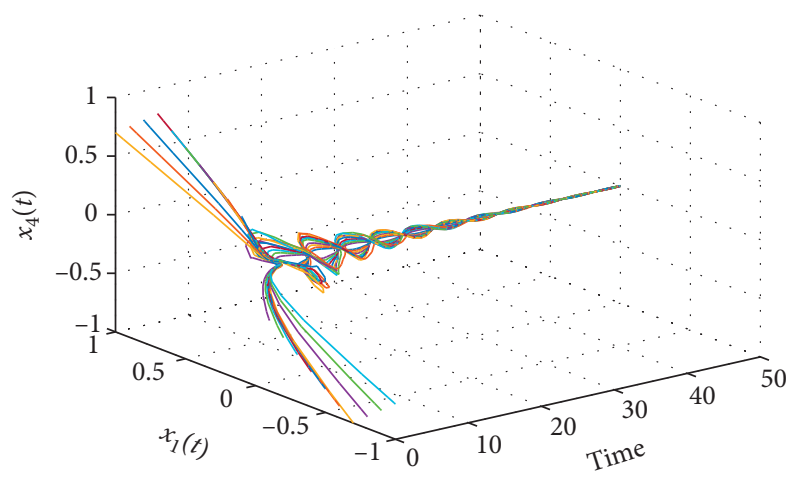

Figure 7: State trajectories $x_{1}(t)$ and $x_{4}(t)$ for $\mu=0.1$ and $h=4.2290$.

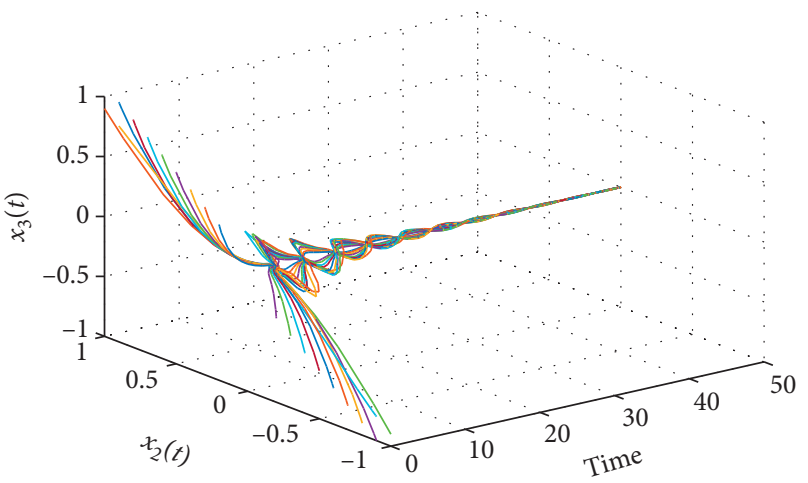

Figure 8: State trajectories $x_{2}(t)$ and $x_{3}(t)$ for $\mu=0.1$ and $h=4.2290$.

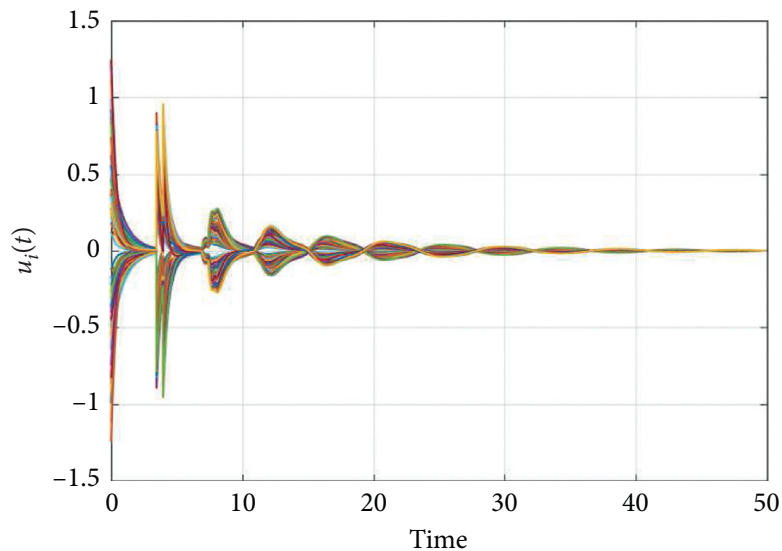

FIGURE 9: Control input of the considered system.

\section{Conclusion}

This study has proposed further results for the stability analysis issue of UDNNs based on the RMFC scheme. First, an improved quadratic function method has been introduced for constructing a novel $V_{1}(x(t))$, which can fully excavate some intrinsic relationships between the delay derivative information and the time delay. Based on the TDPF and LCCM, the information storage has been further improved for obtaining new theoretical results. Second, by using resultful integral inequalities and correlation analysis approaches, several relaxed criteria have been established with respect to the asymptotical stability of the considered UDNNs. Third, a new RMFC has been designed, which can ensure the system stability of UDNNs. Lastly, two numerical experiments have been given to illustrate the significance of the theoretical results. In the future research, we need to further study the UDNNs based on quantized measurements 
in order to improve the entire system [41]. In addition, the results obtained in this paper will be extended to the chaotic Lurie system $[42,43]$, quaternion-valued or memristor-based neural networks [44-46], T-S fuzzy NNs [47, 48], Markov jump systems $[49,50]$, and complex dynamical networks [51-53]. These will occur in the near future.

\section{Appendix}

\section{A. Some Relevant Notations}

$$
\begin{aligned}
& v_{1}(t)=\int_{t-h(t)}^{t} \frac{x^{T}(s)}{h(t)} \mathrm{d} s \\
& \nu_{2}(t)=\int_{t-h}^{t-h(t)} \frac{x^{T}(s)}{h-h(t)} \mathrm{d} s, \\
& v_{3}(t)=\int_{t-h(t)}^{t} \int_{\theta}^{t} \frac{x^{T}(s)}{h^{2}(t)} \mathrm{d} s \mathrm{~d} \theta \\
& v_{4}(t)=\int_{t-h}^{t} \int_{\theta}^{t-h(t)} \frac{x^{T}(s)}{(h-h(t))^{2}} \mathrm{~d} s \mathrm{~d} \theta \\
& \alpha(t)=\operatorname{col}\left\{x(t), x(t-h), h \nu_{1}(t),(h-h(t)) \nu_{2}(t)\right\}, \\
& \eta(t)=\operatorname{col}\{x(t), x(t-h(t)), x(t-h)\}, \\
& \xi(t)=\operatorname{col}\left\{\begin{array}{c}
x(t), x(t-h(t)), x(t-h), \dot{x}(t), \dot{x}(t-h(t)), \dot{x}(t-h), \\
f(x(t)), f(x(t-h(t))), v_{1}(t), v_{2}(t), v_{3}(t), v_{4}(t)
\end{array}\right\}, \\
& \mathscr{P}_{h(t)}=\mathscr{P}_{1}-(h-h(t)) \mathscr{P}_{2},
\end{aligned}
$$

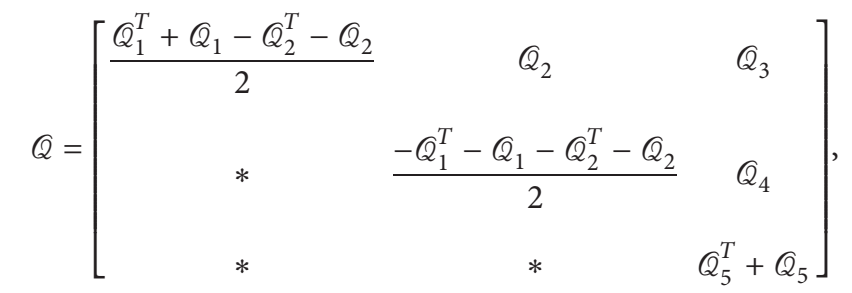

$$
\begin{aligned}
& \mathscr{M}_{1, h(t)}=h(t) \mathscr{M}_{1}+\mathscr{M}_{2} \text {, } \\
& \mathscr{M}_{2, h(t)}=(h-h(t)) \mathscr{M}_{3}+\mathscr{M}_{4} \text {. }
\end{aligned}
$$

\section{B. Related Equations of Theorem 1}

The appendix lists some notations and equations given in

Theorem 1: 


$$
\begin{aligned}
& \Sigma_{h(t), \dot{h}(t)}=\sum_{i=1}^{4} \Xi_{i}+\operatorname{Sym}\{\mathscr{L} \varphi\}, \\
& \Sigma_{1}=\Sigma_{h(t)=0, \dot{h}(t)=0}, \\
& \Sigma_{2}=\Sigma_{h(t)=h, \dot{h}(t)=0}, \\
& \Sigma_{3}=\Sigma_{h(t)=h, \dot{h}(t)=\mu}, \\
& \Sigma_{4}=\Sigma_{h(t)=0, \dot{h}(t)=\mu}, \\
& \Xi_{1}=\dot{h}(t) \Phi_{1}^{T}(h(t)) \mathscr{P}_{2} \Phi_{1}(h(t))+\operatorname{Sym}\left\{\Phi_{1}^{T}(h(t)) \mathscr{P}_{h(t)} \Phi_{2}(\dot{h}(t))\right\}, \\
& \Xi_{2}=\Phi_{3}^{T} Q \Phi_{4}(\dot{h}(t)) \\
& \Xi_{3}=e_{4}^{T}\left[\mathscr{G}_{h(t)}+\mathscr{M}_{1, h(t)}\right] e_{4}+(1-\dot{h}(t)) e_{5}^{T}\left[\mathscr{M}_{2, h(t)}-\mathscr{M}_{1, h(t)}\right] e_{5}-e_{6}^{T} \mathscr{M}_{2, h(t)} e_{6}+\Omega(h(t)) \text {, } \\
& \Xi_{4}=2(1-\dot{h}(t)) e_{8}^{T}\left(\mathscr{D}_{1}-\mathscr{D}_{2}\right) e_{5}+2(1-\dot{h}(t)) e_{2}\left(\mathscr{D}_{2} \mathscr{L}_{2}-\mathscr{D}_{1} \mathscr{L}_{1}\right) e_{5}, \\
& \Gamma(h(t))=\frac{h-h(t)}{h^{2}} \mathscr{E}_{1}^{T} \mathcal{U}_{2}^{T} \Pi_{2, \dot{h}(t)}^{-1} \mathcal{U}_{2} \mathscr{E}_{1}+\frac{h(t)}{h^{2}} \mathscr{E}_{2}^{T} \mathcal{U}_{1}^{T} \Pi_{1}^{-1, \dot{h}(t)} \mathcal{U}_{1} \mathscr{E}_{2}, \\
& \Omega(h(t))=-\frac{1}{h}\left(\frac{2 h-h(t)}{h} \mathscr{E}_{1}^{T} \Pi_{1, \dot{h}(t)} \mathscr{E}_{1}+\frac{h-h(t)}{h} \mathscr{E}_{2}^{T} \mathscr{U}_{1}^{T} \mathscr{E}_{1}+\frac{h-h(t)}{h} \mathscr{E}_{2}^{T} \mathscr{U}_{2}^{T} \mathscr{E}_{1}\right. \\
& \left.+\frac{h-h(t)}{h} \mathscr{E}_{1}^{T} \mathscr{U}_{1} \mathscr{E}_{2}+\frac{h-h(t)}{h} \mathscr{E}_{1}^{T} \mathcal{U}_{2} \mathscr{E}_{2}+\frac{h-h(t)}{h} \mathscr{E}_{2}^{T} \Pi_{2, \dot{h}(t)} \mathscr{E}_{2}\right), \\
& \mathscr{G}_{h(t)}=h(t) \mathscr{G}_{1}+(h-h(t)) \mathscr{G}_{2} \text {, } \\
& \mathscr{L}=e_{1}^{T} \mathcal{N}_{1}+e_{4}^{T} \mathcal{N}_{2}, \\
& \varphi=\left(-\mathscr{A}+(\mathscr{I}-\Pi) \mathscr{K}_{1}\right) e_{1}+\mathscr{W}_{0} e_{7}+\mathscr{W}_{1} e_{8}+(\mathscr{I}-\Pi) \mathscr{K}_{2} e_{2}+(\mathscr{I}-\Pi) \mathscr{K}_{3} e_{3}-e_{4}, \\
& \Gamma=\mathscr{L} \mathscr{H}, \\
& \Theta=\mathscr{E} e_{1} \text {, } \\
& \Phi_{1}(h(t))=\operatorname{col}\left\{e_{1}, e_{3}, h(t) e_{9},(h-h(t)) e_{10}\right\}, \\
& \Phi_{2}(\dot{h}(t))=\operatorname{col}\left\{e_{4}, e_{6}, e_{1}-(1-\dot{h}(t)) e_{2},(1-\dot{h}(t)) e_{2}-e_{3}\right\}, \\
& \Phi_{3}=\operatorname{col}\left\{e_{1}, e_{2}, e_{3}\right\}, \\
& \Phi_{4}(\dot{h}(t))=\operatorname{col}\left\{e_{4},(1-\dot{h}(t)) e_{5}-e_{6}\right\} \text {, } \\
& \mathscr{E}_{1}=\operatorname{col}\left\{e_{1}-e_{2}, e_{1}+e_{2}-2 e_{9}, e_{1}-e_{2}+6 e_{9}-12 e_{11}\right\}, \\
& \mathscr{E}_{2}=\operatorname{col}\left\{e_{2}-e_{3}, e_{2}+e_{3}-2 e_{10}, e_{2}-e_{3}+6 e_{10}-12 e_{12}\right\}, \\
& \bar{\Pi}_{1, \dot{h}(t)}=(1-\dot{h}(t)) \mathscr{G}_{1}+\dot{h}(t) \mathscr{G}_{2}-\dot{h}(t) \mathscr{M}_{1} \text {, } \\
& \bar{\Pi}_{2, \dot{h}(t)}=\mathscr{G}_{2}+\dot{h}(t) \mathscr{M}_{3} \text {, } \\
& e_{i}=\left[\begin{array}{lll}
0_{n \times(i-1) n} & I_{n \times n} & 0_{n \times(9-i)}
\end{array}\right], \quad i=1,2, \ldots, 12, \\
& \Pi_{1, \dot{h}(t)}=\left[\begin{array}{ccc}
\bar{\Pi}_{1, \dot{h}(t)} & 0 & 0 \\
0 & 3 \bar{\Pi}_{1, \dot{h}(t)} & 0 \\
0 & 0 & 12 \bar{\Pi}_{1, \dot{h}(t)}
\end{array}\right] \text {, } \\
& \Pi_{2, \dot{h}(t)}=\left[\begin{array}{ccc}
\bar{\Pi}_{2, \dot{h}(t)} & 0 & 0 \\
0 & 3 \bar{\Pi}_{2, \dot{h}(t)} & 0 \\
0 & 0 & 12 \bar{\Pi}_{2, \dot{h}(t)}
\end{array}\right]
\end{aligned}
$$




\section{Related Equations of Theorem 2}

The appendix lists some notations and equations given in Theorem 2:

$$
\begin{aligned}
& \widetilde{\Sigma}_{h(t), \dot{h}(t)}=\sum_{i=1}^{4} \widetilde{\Xi}_{i}+\operatorname{Sym}\{\widetilde{\mathscr{L}} \varphi\} \\
& \widetilde{\Sigma}_{1}=\widetilde{\Sigma}_{h(t)=0, \dot{h}(t)=0}, \\
& \widetilde{\Sigma}_{2}=\widetilde{\Sigma}_{h(t)=h, \dot{h}(t)=0}, \\
& \widetilde{\Sigma}_{3}=\widetilde{\Sigma}_{h(t)=h, \dot{h}(t)=\mu}, \\
& \widetilde{\Sigma}_{4}=\widetilde{\Sigma}_{h(t)=0, \dot{h}(t)=\mu}, \\
& \widetilde{\Xi}_{1}=\dot{h}(t) \Phi_{1}^{T}(h(t)) \widetilde{\mathscr{P}}_{2} \Phi_{1}(h(t))+\operatorname{Sym}\left\{\Phi_{1}^{T}(h(t)) \widetilde{\mathscr{P}}_{h(t)} \Phi_{2}(\dot{h}(t))\right\} \\
& \widetilde{\Xi}_{2}=\Phi_{3}^{T} \widetilde{Q} \Phi_{4}(\dot{h}(t)), \\
& \widetilde{\Xi}_{3}=e_{4}^{T}\left[\widetilde{\mathscr{G}}_{h(t)}+\widetilde{\mathscr{M}}_{1, h(t)}\right] e_{4}+(1-\dot{h}(t)) e_{5}^{T}\left[\widetilde{\mathscr{M}}_{2, h(t)}-\widetilde{\mathscr{M}}_{1, h(t)}\right] e_{5}-e_{6}^{T} \widetilde{\mathscr{M}}_{2, h(t)} e_{6}+\widetilde{\Omega}(h(t)), \\
& \widetilde{\Xi}_{4}=2(1-\dot{h}(t)) e_{8}^{T}\left(\tilde{\mathscr{D}}_{1}-\tilde{\mathscr{D}}_{2}\right) e_{5}+2(1-\dot{h}(t)) e_{2}\left(\tilde{\mathscr{D}}_{2} \mathscr{L}_{2}-\tilde{\mathscr{D}}_{1} \mathscr{L}_{1}\right) e_{5}, \\
& \widetilde{\Gamma}(h(t))=\frac{h-h(t)}{h^{2}} \mathscr{E}_{1}^{T} \widetilde{\mathcal{U}}_{2}^{T} \widetilde{\Pi}_{2, \dot{h}(t)}^{-1} \widetilde{\mathcal{U}}_{2} \mathscr{E}_{1}+\frac{h(t)}{h^{2}} \mathscr{E}_{2}^{T} \widetilde{\mathcal{U}}_{1}^{T} \widetilde{\Pi}_{1}^{-1, \dot{h}(t)} \widetilde{\mathcal{U}}_{1} \mathrm{E}_{2}, \\
& \widetilde{\Omega}(h(t))=-\frac{1}{h}\left(\frac{2 h-h(t)}{h} \mathscr{E}_{1}^{T} \widetilde{\Pi}_{1, \dot{h}(t)} \mathscr{E}_{1}+\frac{h-h(t)}{h} \mathscr{E}_{2}^{T} \widetilde{\mathcal{U}}_{1}^{T} \mathscr{E}_{1}+\frac{h-h(t)}{h} \mathscr{E}_{2}^{T} \widetilde{\mathcal{U}}_{2}^{T} \mathscr{E}_{1}\right. \\
& \left.+\frac{h-h(t)}{h} \mathscr{E}_{1}^{T} \widetilde{\mathcal{U}}_{1} \mathscr{E}_{2}+\frac{h-h(t)}{h} \mathscr{E}_{1}^{T} \widetilde{\mathscr{U}}_{2} \mathscr{E}_{2}+\frac{h-h(t)}{h} \mathscr{E}_{2}^{T} \widetilde{\Pi}_{2, \dot{h}(t)} \mathscr{E}_{2}\right) \text {, } \\
& \widetilde{\mathscr{G}}_{h(t)}=h(t) \widetilde{\mathscr{G}}_{1}+(h-h(t)) \widetilde{\mathscr{G}}_{2} \text {, } \\
& \widetilde{\mathscr{L}}=e_{1}^{T}+\kappa e_{4}^{T} \text {, } \\
& \widetilde{\varphi}=\left(-\mathscr{L} \mathscr{H}+(\mathscr{I}-\Pi) \mathscr{Y}_{1}\right) e_{1}+\mathscr{W}_{0} \mathscr{V} e_{7}+\mathscr{W}_{1} \mathscr{V} e_{8}+(\mathscr{I}-\Pi) \mathscr{Y}_{2} e_{2}+(\mathscr{I}-\Pi) \mathscr{Y}_{3} e_{3}-e_{4} \mathscr{V}, \\
& \widetilde{\Gamma}=\widetilde{\mathscr{L}} \mathscr{H}, \\
& \widetilde{\Theta}=\mathrm{E} e_{1}, \\
& \widetilde{\bar{\Pi}}_{1, \dot{h}(t)}=(1-\dot{h}(t)) \widetilde{\mathscr{G}}_{1}+\dot{h}(t) \widetilde{\mathscr{G}}_{2}-\dot{h}(t) \widetilde{\mathscr{M}}_{1} \text {, } \\
& \widetilde{\Pi}_{2, \dot{h}(t)}=\widetilde{\mathscr{G}}_{2}+\dot{h}(t) \widetilde{\mathscr{M}}_{3} \text {, } \\
& \widetilde{\Pi}_{1, \dot{h}(t)}=\left[\begin{array}{ccc}
\widetilde{\bar{\Pi}}_{1, \dot{h}(t)} & 0 & 0 \\
0 & 3 \widetilde{\bar{\Pi}}_{1, \dot{h}(t)} & 0 \\
0 & 0 & 12 \widetilde{\bar{\Pi}}_{1, \dot{h}(t)}
\end{array}\right] \text {, } \\
& \widetilde{\Pi}_{2, \dot{h}(t)}=\left[\begin{array}{ccc}
\tilde{\bar{\Pi}}_{2, \dot{h}(t)} & 0 & 0 \\
0 & 3 \widetilde{\bar{\Pi}}_{2, \dot{h}(t)} & 0 \\
0 & 0 & 12 \widetilde{\bar{\Pi}}_{2, \dot{h}(t)}
\end{array}\right] \text {. }
\end{aligned}
$$




\section{Data Availability}

The raw/processed data required to reproduce these findings cannot be shared at this time as the data also form part of an ongoing study.

\section{Disclosure}

The authors declare that the work described is original research that has not been published previously and not under consideration for publication elsewhere, in whole or in part.

\section{Conflicts of Interest}

The authors declare no conflicts of interest.

\section{Authors' Contributions}

All the authors listed approved the manuscript that is enclosed.

\section{References}

[1] L. O. Chua and L. Yang, "Cellular neural networks: applications," IEEE Transactions on Circuits and Systems, vol. 35, no. 10, pp. 1273-1290, 1988.

[2] S. Arik, "An analysis of exponential stability of delayed neural networks with time varying delays," Neural Networks, vol. 17, no. 7, pp. 1027-1031, 2004.

[3] D. Ding, Z. Wang, Q.-L. Han, and G. Wei, "Neural-Networkbased output-feedback control under round-robin scheduling protocols," IEEE Transactions on Cybernetics, vol. 49, no. 6 , pp. 2372-2384, 2019.

[4] W. B. Liu, Z. D. Wang, X. H. Liu, N. Y. Zeng, Y. R. Liu, and F. E. Alsaadi, "A survey of deep neural network architectures and their applications," Neurocomputing, vol. 234, pp. 11-26, 2017.

[5] X. Zhang, X. Li, J. Cao, and F. Miaadi, "Design of memory controllers for finite-time stabilization of delayed neural networks with uncertainty," Journal of the Franklin Institute, vol. 355, no. 13, pp. 5394-5413, 2018.

[6] J.-N. Li, Y. Zhang, and Y.-J. Pan, "Mean-square exponential stability and stabilisation of stochastic singular systems with multiple time-varying delays," Circuits, Systems, and Signal Processing, vol. 34, no. 4, pp. 1187-1210, 2015.

[7] O. M. Kwon, M. J. Park, S. M. Lee, J. H. Park, and E. J. Cha, "Stability for neural networks with time-varying delays via some new approaches," IEEE Transactions on Neural Networks and Learning Systems, vol. 24, no. 2, pp. 181-193, 2013.

[8] S. Amari and A. Cichocki, "Adaptive blind signal processingneural network approaches," Proceedings of the IEEE, vol. 86, pp. 2026-2048, 1998.

[9] M. Aminian and F. Aminian, "Neural-network based analog-circuit fault diagnosis using wavelet transform as preprocessor," IEEE Transactions on Circuits and Systems II: Analog and Digital Signal Processing, vol. 47, no. 2, pp. 151-156, 2000.

[10] M. Mestari, M. Benzirar, N. Saber, and M. Khouil, "Solving nonlinear equality constrained multiobjective optimization problems using neural networks," IEEE Transactions on Neural Networks and Learning Systems, vol. 26, no. 10, pp. 2500-2520, 2015.
[11] J. K. Tian, S. M. Zhong, and Y. Wang, "Hierarchical singleton-type recurrent neural fuzzy networks for noisy speech recognition," IEEE Transactions on Neural Networks, vol. 18, pp. 833-843, 2007.

[12] G. Costantini, D. Casali, and R. Perfetti, “Associative memory design for 256 gray-level images using a multilayer neural network," IEEE Transactions on Neural Networks, vol. 17, no. 2, pp. 519-522, 2006.

[13] H. Zhang, Z. Liu, G. B. Huang, and Z. Wang, "Novel weighting-delay-based stability criteria for recurrent neural networks with time-varying delay," IEEE Transactions on Neural Networks, vol. 21, no. 1, pp. 91-106, 2010.

[14] S. P. Xiao and X. M. Zhang, "New globally asymptoic stability criteria for delayed cellular neural networks," IEEE Transactions on Circuits and Systems II: Express Briefs, vol. 56, pp. 659-663, 2009.

[15] X. M. Zhang and Q. L. Han, "New Lyapunov-krasovskii functionals for global asymptotic stability of delayed neural networks," IEEE Transactions on Neural Networks, vol. 20, no. 3, pp. 533-539, 2009.

[16] H. B. Zeng, Y. He, M. Wu, and C. F. Zhang, "Complete delay-decomposing approach to asymptotic stability for neural networks with time-varying delays," IEEE Transactions on Neural Networks, vol. 22, no. 5, pp. 806-812, 2011.

[17] G. Cao, C. Hua, and X. Guan, "New delay-dependent stability criteria for neural networks with time-varying delay using delay-decomposition approach," IEEE Transactions on Neural Networks and Learning Systems, vol. 25, pp. 1378-1383, 2014.

[18] H.-B. Zeng, Y. He, M. Wu, and S.-P. Xiao, "Stability analysis of generalized neural networks with time-varying delays via a new integral inequality," Neurocomputing, vol. 161, pp. 148-154, 2015.

[19] C.-K. Zhang, Y. He, L. Jiang, Q. H. Wu, and M. Wu, "Delay-dependent stability criteria for generalized neural networks with two delay components," IEEE Transactions on Neural Networks and Learning Systems, vol. 25, no. 7, pp. 1263-1276, 2014.

[20] Y. Liu, S. M. Lee, O. M. Kwon, and J. H. Park, "New approach to stability criteria for generalized neural networks with interval time-varying delays," Neurocomputing, vol. 149, pp. 1544-1551, 2015.

[21] R. Manivannan, G. Mahendrakumar, R. Samidurai, J. Cao, and A. Alsaedi, "Exponential stability and extended dissipativity criteria for generalized neural networks with interval time-varying delay signals," Journal of the Franklin Institute, vol. 354, no. 11, pp. 4353-4376, 2017.

[22] J. Wang, K. Shi, Q. Huang, S. Zhong, and D. Zhang, "Stochastic switched sampled-data control for synchronization of delayed chaotic neural networks with packet dropout," Applied Mathematics and Computation, vol. 335, pp. 211-230, 2018.

[23] X. M. Zhang and Q. L. Han, "Global asymptotic stability for a class of generalized neural networks with interval time-varying delays," IEEE Transactions on Neural Networks, vol. 22, no. 8, pp. 1180-1192, 2011.

[24] R. Manivannan, R. Samidurai, J. Cao, A. Alsaedi, and F. E. Alsaadi, "Global exponential stability and dissipativity of generalized neural networks with time-varying delay signals," Neural Networks, vol. 87, pp. 149-159, 2017.

[25] X. Cai, S. Zhong, J. Wang, and K. Shi, "New stability results for delayed neural networks with data packet dropouts," Physica A: Statistical Mechanics and Its Applications, vol. 555, Article ID 124727, 2020. 
[26] G. H. Yang and D. Ye, "Reliable $H_{\infty}$ control of linear systems with adaptive mechanism," IEEE Transactions on Automatic Control, vol. 55, no. 1, pp. 242-247, 2010.

[27] D. Ye, L. Su, J.-L. Wang, and Y.-N. Pan, "Adaptive reliable \$H_\{linfty \}\$ optimization control for linear systems with time-varying actuator fault and delays," IEEE Transactions on Systems, Man, and Cybernetics: Systems, vol. 47, no. 7, pp. 1635-1643, 2017.

[28] K. Shi, J. Wang, S. Zhong, X. Zhang, Y. Liu, and J. Cheng, "New reliable nonuniform sampling control for uncertain chaotic neural networks under Markov switching topologies," Applied Mathematics and Computation, vol. 347, pp. 169-193, 2019.

[29] H. Li, H. Liu, H. Gao, and P. Shi, "Reliable fuzzy control for active suspension systems with actuator delay and fault," IEEE Transactions on Fuzzy Systems, vol. 20, no. 2, pp. 342-357, 2012.

[30] B. Yang, M. Hao, J. Cao, and X. Zhao, "Delay-dependent global exponential stability for neural networks with time-varying delay," Neurocomputing, vol. 338, pp. 172-180, 2019.

[31] C.-K. Zhang, Y. He, L. Jiang, M. Wu, and Q.-G. Wang, “An extended reciprocally convex matrix inequality for stability analysis of systems with time-varying delay," Automatica, vol. 85, pp. 481-485, 2017.

[32] X.-M. Zhang, Q.-L. Han, A. Seuret, and F. Gouaisbaut, "An improved reciprocally convex inequality and an augmented lyapunov-krasovskii functional for stability of linear systems with time-varying delay," Automatica, vol. 84, pp. 221-226, 2017.

[33] H.-B. Zeng, Y. He, M. Wu, and J. She, "Free-matrix-based integral inequality for stability analysis of systems with time-varying delay," IEEE Transactions on Automatic Control, vol. 60, no. 10, pp. 2768-2772, 2015.

[34] L. Zhi, Y. He, C. K. Zhang, and M. Wu, "Robust $H_{\infty}$ control for T-S fuzzy systems with state and input time-varying delays via delay-product-type functional method," IEEE Transactions on Fuzzy Systems, vol. 27, no. 10, pp. 1917-1930, 2019.

[35] H.-B. Zeng, X.-G. Liu, and W. Wang, "A generalized freematrix-based integral inequality for stability analysis of time-varying delay systems," Applied Mathematics and Computation, vol. 354, pp. 1-8, 2019.

[36] X. Cai, J. Wang, S. M. Zhong, K. B. Shi, and Y. Q. Tang, "Fuzzy quantized sampled-data control for extended dissipative analysis of T-S fuzzy system and its application to WPGSs," Journal of the Franklin Institute, vol. 358, no. 2, 2020.

[37] H.-B. Zeng, K. L. Teo, and Y. He, "A new looped-functional for stability analysis of sampled-data systems," Automatica, vol. 82, pp. 328-331, 2017.

[38] Y. Yuan, F. Sun, and Q. Zhu, "Resilient control in the presence of dos attack: switched system approach," International Journal of Control, Automation and Systems, vol. 13, no. 6, pp. 1423-1435, 2015.

[39] Y. He, G. Liu, and D. Ree, "Exponential stability on stochastic neural networks with discrete interval and distributed delays," IEEE Transactions on Neural Networks, vol. 21, pp. 169-175, 2010.

[40] X. Cai, S. Zhong, J. Wang, and K. Shi, "Robust Hoo control for uncertain delayed T-S fuzzy systems with stochastic packet dropouts," Applied Mathematics and Computation, vol. 385, Article ID 125432, 2020.

[41] X. Cai, K. Shi, S. Zhong, and X. Pang, "Dissipative sampled-data control for high-speed train systems with quantized measurements," IEEE Transactions on Intelligent Transportation Systems, vol. 99, pp. 1-12, 2021.
[42] K. B. Shi, Y. Y. Tang, S. M. Zhong, C. Yin, X. G. Huang, and W. Q. Wang, "Nonfragile asynchronous control for uncertain chaotic Lurie network systems with Bernoulli stochastic process," International Journal of Robust and Nonlinear Control, vol. 28, no. 5, pp. 1693-1714, 2018.

[43] K. Shi, Y. Tang, X. Liu, and S. Zhong, "Non-fragile sampled-data robust synchronization of uncertain delayed chaotic Lurie systems with randomly occurring controller gain fluctuation," ISA Transactions, vol. 66, pp. 185-199, 2017.

[44] L. Hua, S. Zhong, K. Shi, and X. Zhang, "Further results on finite-time synchronization of delayed inertial memristive neural networks via a novel analysis method," Neural Networks, vol. 127, pp. 47-57, 2020.

[45] X. R. Ji, J. Q. Lu, J. G. Lou, J. L. Qiu, and K. B. Shi, “A unified criterion for global exponential stability of quaternion-valued neural networks with hybrid impulses," International Journal of Robust and Nonlinear Control, vol. 30, no. 18, pp. 8098-8116, 2020.

[46] L. Hua, H. Zhu, K. Shi, S. Zhong, Y. Tang, and Y. Liu, "Novel finite-time reliable control design for memristor-based inertial neural networks with mixed time-varying delays," IEEE Transactions on Circuits and Systems I: Regular Papers, pp. 1-11, 2021.

[47] K. B. Shi, J. Wang, Y. Y. Tang, and S. M. Zhong, "Reliable asynchronous sampled-data filtering of TCS fuzzy uncertain delayed neural networks with stochastic switched topologies," Fuzzy Sets and Systems, vol. 381, pp. 1-25, 2020.

[48] K. Shi, J. Wang, S. Zhong, Y. Tang, and J. Cheng, "Non-fragile memory filtering of T-S fuzzy delayed neural networks based on switched fuzzy sampled-data control," Fuzzy Sets and Systems, vol. 394, pp. 40-64, 2020.

[49] C. Zhao, S. M. Zhong, Q. S. Zhong, and K. B. Shi, "Synchronization of Markovian complex networks with input mode delay and Markovian directed communication via distributed dynamic event-triggered control," Nonlinear Analysis: Hybrid Systems, vol. 36, Article ID 100883, 2020.

[50] W. Xie, R. Zhang, D. Zeng, K. Shi, and S. Zhong, "Strictly dissipative stabilization of multiple-memory Markov jump systems with general transition rates: a novel event-triggered control strategy," International Journal of Robust and Nonlinear Control, vol. 30, no. 5, pp. 1956-1978, 2020.

[51] K. Shi, J. Wang, S. Zhong, Y. Tang, and J. Cheng, "Hybrid-driven finite-time Hoo sampling synchronization control for coupling memory complex networks with stochastic cyber attacks," Neurocomputing, vol. 387, pp. 241-254, 2020.

[52] Y. D. Wang, X. H. Hu, K. B. Shi, X. N. Song, and H. Shen, "Network-based passive estimation for switched complex dynamical networks under persistent dwell-time with limited signals," Journal of the Franklin Institute, vol. 357, no. 15, pp. 10921-10936, 2020.

[53] C. Zhao, S. M. Zhong, X. J. Zhang, Q. S. Zhong, and K. B. Shi, "Novel results on nonfragile sampled-data exponential synchronization for delayed complex dynamical networks," Nternational Journal of Robust and Nonlinear Control, vol. 30, no. 9, pp. 4022-4042, 2020. 